\title{
Multilayer Silicon RF System-in-Package Technique Using Magnetically Aligned Anisotropic Conductive Adhesive
}

\author{
Sungwook Moon ${ }^{1}$, S. Kummar Khanna ${ }^{2}$, and William J. Chappell ${ }^{1}$ \\ ${ }^{1}$ Purdue University, School of Electrical and Computer Engineering, West Lafayette, IN, 47907, USA \\ ${ }^{2}$ Shiva Consulting Inc., Carmel, IN, 46033, USA
}

\begin{abstract}
In this work, we propose a novel packaging concept for highly-integrated RF systems using a magnetically aligned Zaxis anisotropic conductive adhesive. We demonstrate the ability to "grow" interconnects allowing for multilayer packages that are not sensitive to the height between pads. Using this effect we introduce two approaches to integrating multiple silicon wafers on top of each other, creating the possibility for an exceptionally dense integrated system-in-a-package. First, a reverse-pyramid package with all chips stacked facing down on a silicon substrate is demonstrated. Second, a "Matryoshka" package assembled with the alternation of chip's face direction is also demonstrated. The simplified assembly process of the Z-axis ACA and the new packaging concepts can offer a compact and cost-effective solution to system-in-package based RF systems.
\end{abstract}

Index Terms - RF Packaging, System-in-package, multi-chip stacking, anisotropic conductive adhesive, vertical interconnects.

\section{INTRODUCTION}

Today, 3-D packaging technology is required to satisfy various requirements for applications as varied as wireless biomedical applications [1], high density array antenna applications [2], to handheld consumer electronics [3] including multi-chip stacked memories and high-speed stacked processors. The 2007 International Technology Roadmap for Semiconductors (ITRS) indicates that 3-D advanced packaging technology based on wafer-level packaging must move toward developing processes for larger die and higher functionality applications. These applications include system-in-package (SiP) with chip to wafer and 3-D configurations, multiple IC stacks, and wafer-to-wafer stacks [3]. A growing interest concerning chip interconnection techniques to support 3-D packaging technology has developed since the quality of these interconnects has a large impact on the performance of the entire system, especially at high frequencies [4]. Already popular for memory applications, multiple stacked die traditionally require complex multi-level wirebonding. This technique is particularly problematic for high frequency applications where loop height and pad pitch must be minimized to decrease the induced inductances of the long wirebonds [5]. Another technology, the bumping approach, has been developed based on flip-chip packaging technology and its RF performance for integrated and $\mathrm{mm}-$ wave applications were studied in [6]. However, this approach is not currently applicable for more than two layers of silicon, or is at least very difficult because of the need for different balls and the balancing of stresses during the process. In

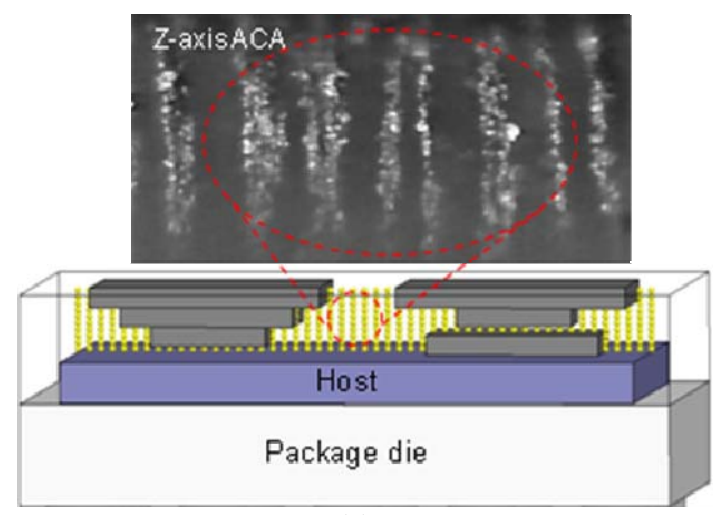

(a)



(b)

Fig. 1. (a) Schematic of system-in-package based on a magnetically aligned Z-axis ACA and (b) created conductive columns in a $\mathrm{Z}$-axis ACA, and equivalent lumped model of created vertical interconnects in an overlapped pad.

addition, the fabrication process of the bumping approach is complicated and the RF performance of the interconnects is sensitive to bump height, diameter, and bump to bump spacing.

In this work, we discuss a polymer-based packaging approach that can integrate multiple layers of silicon which has advantages such as a thin form factor, assembly cost and process-reduction, environmental friendliness (such as no lead), and low parasitic impedance. For high frequency applications, this is particularly relevant because of the short interconnects needed while providing the integration of multiple layers of silicon on silicon. Multi-layer interconnects are demonstrated using a flip-chip like approach with a novel epoxy material that self aligns after epoxy application [7]. By flipping the chips relative to the host, the interconnect lengths are substantially shorter than the face-up wire-bonding approach, leading to much lower parasitic impedances and 


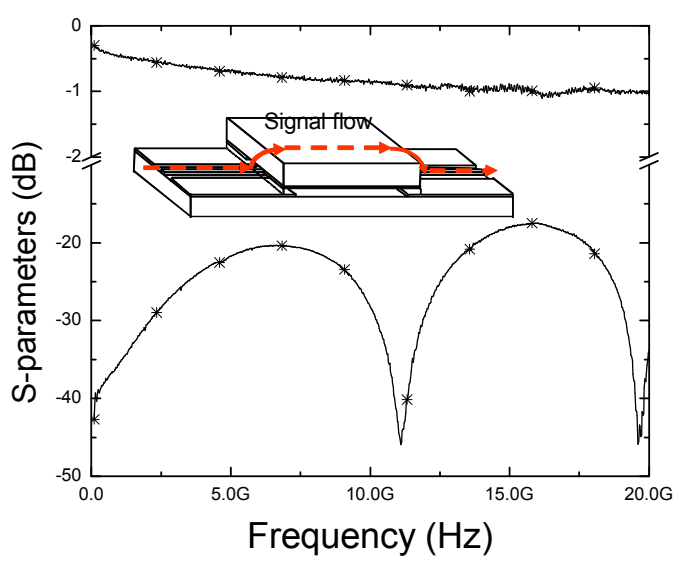

Fig. 2. RF characteristics of a $\mathrm{CPW}$ in a silicon sample assembled with a Z-axis ACA (one-level wafer connection).

opening the door to high frequency applications as shown in Fig. 1 (a). The challenge of this work is creating a material that will allow for multiple layers of interconnects and for this we apply a novel material which will "grow" rods internal to the material to accommodate a range of interconnect heights.

In a $\mathrm{SiP}$ package module, multi-functional chips such as an analog chip, a MEMS chip and a stacked memory module are able to be attached on a digital processor for highly-integrated RF and multifunctional systems. Specifically, for good performance of an integrated RF system at high frequencies, it is important to maintain vertical interconnects with low parasitic impedance. Fig. 1 (b) shows the functionality of a Zaxis ACA, where created columns are vertically connected while isolated between each other as conductive particles are self-aligned in the presence of a magnetic field. The RF performance of the Z-axis ACA up to $90 \mathrm{GHz}$ on silicon substrates were already characterized in our previous work [8], but was only applied for a traditional one-level wafer connection. Fig. 2 shows the RF characteristics of a coplanar waveguide (CPW) of a silicon sample assembled with a Z-axis ACA. The insertion loss of the Z-axis ACA has less than 0.23 $\mathrm{dB}$ of additional loss compared to solder bumps at $20 \mathrm{GHz}$ and the calculated self-resonant frequency caused by parasitic parallel capacitance and inductance is near $110 \mathrm{GHZ}$ in a Zaxis ACA with $50 \mu \mathrm{m}$ thickness. Therefore, the overall high frequency performance of this packaging technique is not limited by the utilization of a Z-axis ACA. The multi-level capabilities that this material enables have never been exploited until now.

\section{IMPLEMENTATION OF MULTI-CHIP PACKAGING}

In order to implement the proposed $\mathrm{SiP}$ using a $\mathrm{Z}$-axis ACA, we propose two novel multi-chip stacking schemes. In this work, we focus on verification of the proposed concepts by showing that interconnects can be created on multiple levels with small pad size $(100 \mu \mathrm{m}$ x $100 \mu \mathrm{m})$. A Z-axis ACA

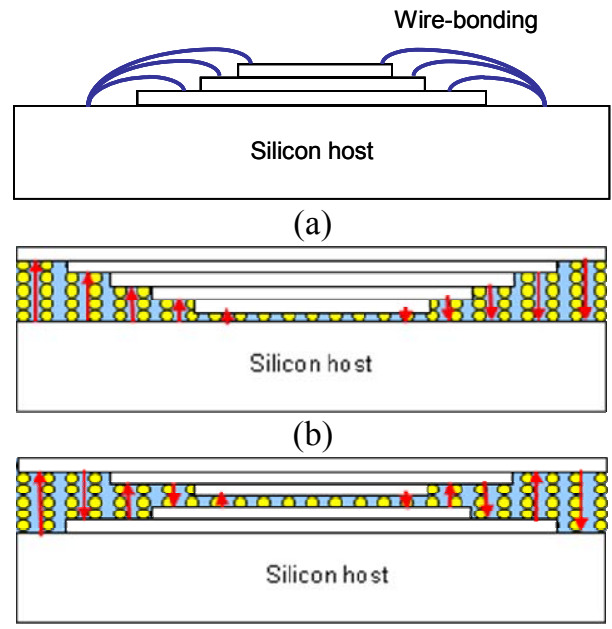

(C)

Fig. 3 (a) Schematic of a conventional pyramid packaging (wire-bonding), (b) concept of reverse-pyramid packaging and (c) Matryoshka packaging concept (Z-axis ACA).

consists of epoxy and conductive particles whose core is $\mathrm{Ni}$ and whose surface is coated with $\mathrm{Au}$ (weight ratio $8 \%$ ) for vertical self-alignment in the presence of a static magnetic field. The Z-axis ACA has spherically shaped nickel cores with an average diameter of $6.6 \mu \mathrm{m}$, which creates columns with $10 \mu \mathrm{m}$ average diameter. The column dimensions are determined from visual inspection using microscopic images of cured Z-axis ACA samples. From top-view images the density of columns is counted to have an average of 977 columns $/ \mathrm{mm}^{2}$.

\section{A. Chip and Host Substrate Fabrication}

A silicon substrate with high resistivity $(10,000 \mathrm{~cm} \cdot \Omega)$ is useful for validating the interconnections of the proposed packaging concepts. The substrate fabrication process for this experiment is as follows; (1) deposition of $\mathrm{Ti}$ and $\mathrm{Au},(2)$ patterning the features (lithography and development), (3) wet etching, (4) dicing wafer, and (5) thinning chips.

In this work, the modules are assembled with three chip layers to verify both the reverse-pyramid and the Matryoshka packaging concepts. The sizes of chips 1,2, and 3 are $3 \mathrm{~mm} \mathrm{x}$ $3 \mathrm{~mm}, 8 \mathrm{~mm} \times 8 \mathrm{~mm}$ and $13 \mathrm{~mm}$ x $13 \mathrm{~mm}$, respectively. The size of the host substrate is $40 \mathrm{~mm}$ x $40 \mathrm{~mm}$ in order to easily measure the pads. The size of overlapping pads for interconnects between chips in all cases is $100 \mu \mathrm{m} \times 100 \mu \mathrm{m}$. The wafers were thinned to below $100 \mu \mathrm{m}$ by a diamond grinding process. When compared with conventional chip stacking technologies such as multi-level wirebonding and bumping, packaging using a magnetically aligned Z-axis ACA is able to remarkably reduce the number of assembly steps in implementing a SiP-based RF system because it does not require any solder bumps and metal layer on the pads for physical contact nor complicated processing to form vias for vertical interconnects. 


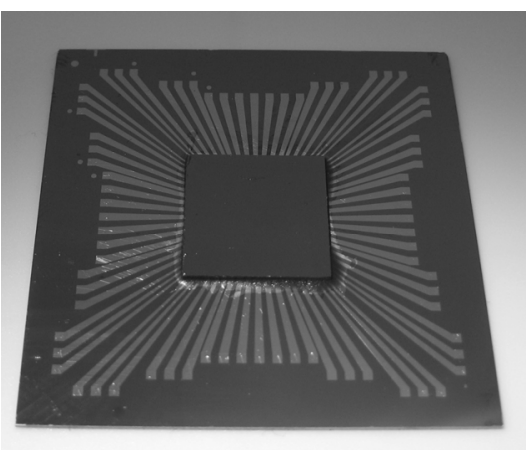

(a)

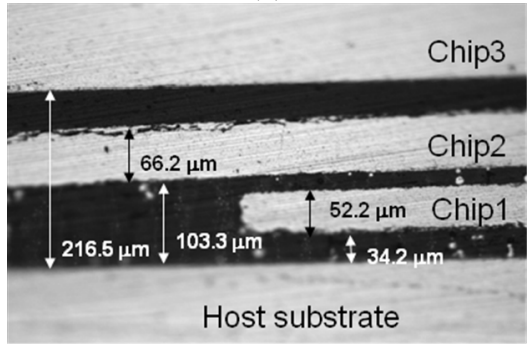

(b)

Fig. 4. (a) Photograph of an assembled module based on a reverse pyramid packaging and (b) its cross-section.

\section{B. Reverse Pyramid Vertical Stacking}

Multiple chips are stacked on a host substrate from smallest to largest to form a novel packaging concept ("reversepyramid") as shown in Fig. 3 (b). In this structure the stacked chips face down toward the host substrate. The length of the created interconnects in a $\mathrm{Z}$-axis ACA is controlled by the separation of stacked chips from the host substrate because the interconnects form dynamically after epoxy application and do not directly set the interconnect length. This "growing" of the rods is in contrast to relying on the pressure applied or the height of the connecting balls as is the case in other approaches. The rods can be created on multiple layers simultaneously and will grow to the height needed and therefore are very forgiving of tolerances in assembly.

It is of interest to discover whether simultaneous or sequential curing process is preferred in the creation of multilayer silicon stacks. The reverse-pyramid packaging technique is used to investigate the performance change of interconnects by curing process order. A serial curing process is carried out as follows; (1) a Z-axis ACA was manually dispensed onto the host substrate, (2) the alignment between a chip (chip 1) and the host substrate is carried out by using a flip-chip bonding process (FINETECH GmbH \& Co, +/- $5 \mu \mathrm{m}$ accuracy), and (3) the assembly sample is then placed in a magnetic oven at $160{ }^{\circ} \mathrm{C}$ for 15 minutes in the presence of a 0.25 tesla magnetic field. The steps of the assembly process from (1) to (3) are repeated until the last chip 3 is stacked on the host substrate. The assembled sample and its cross-section are shown in Fig. 4 (a) and (b).

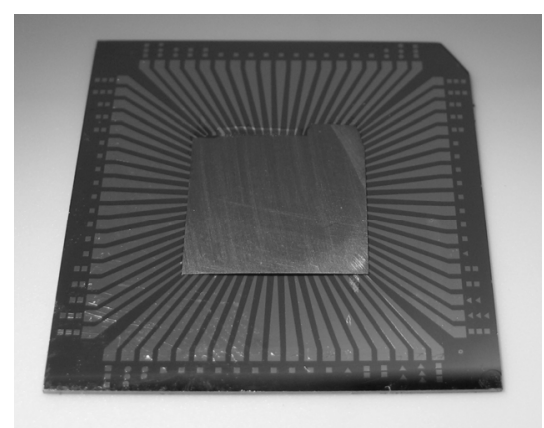

(a)

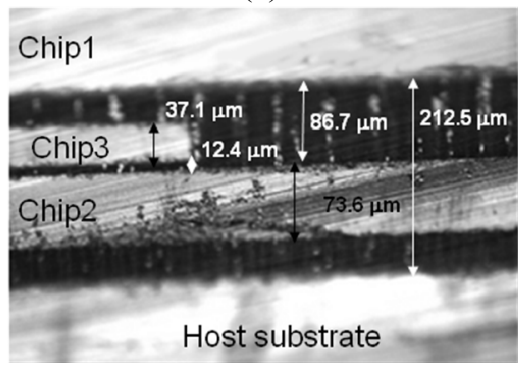

(b)

Fig. 5 (a) Photograph of an assembled module based on Matryoshka packaging and (b) its cross-section.

It is necessary to investigate the influence of an additional curing process on already created interconnects in a cured Zaxis ACA sample. To this end a sample is cured and measured then reheated to $160{ }^{\circ} \mathrm{C}$ for 15 minutes and remeasured. The average resistance changed from $2.9 \Omega$ to 4.8 $\Omega$, and its standard deviation also changed from 0.43 to 1.57 in 18 overlapped pads. From this experimental result, this serial assembly process is not desirable when more than one chip is to be stacked. Instead, a simultaneous curing method is required for stacking multiple chips on a host substrate to prevent the degradation of performance due to repeated exposure to strong magnetic fields and curing cycles.

\section{Nested Chip Stacking}

By alternating the chip-face direction on neighboring chips, as opposed to direct attachment of a chip onto a host substrate, a novel nested packaging concept (or "Matryoshka" design) is built as shown in Fig. 3 (c). While the reverse-pyramid package has direct interconnects from the chips to the host substrate, the electrical paths in this nested "Matryoshka" concept are connected between facing chips in succession. This concept is useful to implement a SiP with several subfunctional chips on a host chip or die as shown in the right part of Fig. 1(a). Z-axis ACA was applied in between each chip between each assembly stage, but curing nor magnetic field was applied until the final assembly was finished. First chip 3 stacks on chip 2, and then the assembled module with the chip 2 and 3 stacks on chip 1. To maintain alignment between chips, an instant curing epoxy is used at the edges of the chips. Finally the three chip- module is flipped over and assembled on a host substrate. The curing process is then 
carried out once. The assembled sample and its cross-section are shown in Fig. 5 (a) and (b). To our knowledge, this is the only method in which interconnects on multiple levels can be formed dynamically.

\section{Measurement and Analysis}

From both packaging features, the reverse-pyramid concept is able to become an alternative to the conventional memory stacking module ("pyramid" approach as shown in Fig. 3 (a)) with wire-bonding technology because it has a more simplified assembly process. In terms of the design of a multifunctional stacked module, the Matryoshka concept is more attractive because it gives the flexibility of optimizing interconnect distribution to provide the shortest signal path between associated chips. This is in contrast to the excess length required if all interconnects go through the host wafer as in the pyramid or reverse-pyramid approaches.

To verify the performance of interconnects in the proposed packaging concepts, the DC resistances of assembled modules are measured by a digital voltage multi-meter. The vertical interconnect resistance represents the overall resistance of a pathway from the pad of a host substrate to the pad of a chip. The resistance of vertical interconnects in a Matryoshka package accumulate all resistances of vertical interconnects located in the signal pathway while the reverse-pyramid package includes the resistance of only one vertical interconnect.

In the reverse-pyramid package, when chip 1 was assembled, the average measured resistance was less than $2 \Omega$ for a $34.2 \mu \mathrm{m}$ length connection. However, after completing the full assembly process (two more temperature cycles to bond chips 2 and 3), the interconnection resistance of the chip 1 was changed to an average of $6.6 \Omega$ in 28 overlapped pads as we discussed earlier in the section B. This is because the repeated temperature cycles to attach successive chips weaken the epoxy binder of the Z-axis ACA allowing the conductive particles to shift resulting in weaker electrical contacts. However, the length of vertical interconnects from the host to chip 3, the final applied chip with no repeated cure cycle, is $216.5 \mu \mathrm{m}$ and their average resistance is $7.0 \Omega$. Also, there exist no open pads of the 24 overlapped pads of the chip 3 with approximately $210 \mu \mathrm{m}$-long gap. This result indicates that conductive particles in a Z-axis ACA are "growing" enough to implement a multi-layer package. The ability to bond over multiple levels and pad-to-pad distances was demonstrated through this experiment, even though curing in one step is found to be beneficial. The balls in the ACA were able to form long chains of interconnects without creating branches and/or shorting with each other.

In the Matryoshka packaging, after the chips were aligned and assembled, the vertical interconnects for all signal pathways were created simultaneously in a single curing process. As a result, the length of vertical interconnects from the host to chip 1 is $212.5 \mu \mathrm{m}$ and their average resistance is $8.9 \Omega$ while the total lengths for chip 2 and 3 are $299.3 \mu \mathrm{m}$ and $311.6 \mu \mathrm{m}$, and their average resistance are $8.7 \Omega$ and 10.2
$\Omega$. Minor misalignment affects the resistance of the chip 1 interconnects more than the other chips, demonstrating the importance of using precise care in alignment. The most significant result is that the created interconnects in a Z-axis ACA work in the pads of all attached chips when a simultaneous curing method is used. Therefore, conductive columns created in a Z-axis ACA using the simultaneous curing method are a novel and useful method for advanced levels of RF integration.

\section{CONCLUSION}

In this work, we demonstrated novel packaging concepts for SiP-based multi-layer silicon RF systems using a magnetically aligned Z-axis ACA. In addition, we analyzed their DC path resistances when both concepts are realized on silicon substrates. As a result, we show that the applied Z-axis ACA works in vertical interconnects for both a reverse-pyramid package with approximately $210 \mu \mathrm{m}$ long gap (chip to host connection), and of a Matryoshka packaging with approximately $310 \mu \mathrm{m}$ long gap (connection between all three chips and the host) between a chip and the host substrate. Therefore, the multilayer packaging application of the Z-axis ACA based on the proposed packaging concepts indicates the potential of a new multi-chip stacking technology for implementing highly-integrated RF SiP modules.

\section{REFERENCES}

[1] E. Chow, et al. "Implantable wireless telemetry boards for in vivo transocular transmission." IEEE Trans. Microwave. Theory Tech., vol. 56, no.12, pp. 3200-3208, Dec. 2008.

[2] K. A. Shalkhouser, "System-level integrated circuits for phased array antenna applications." 1996 IEEE MTT-S Int. Microwave Symp. Dig., pp. 1593-1596, 1996.

[3] International Technology Roadmap for Semiconductor 2007 (ITRS 2007) [Online]. Available: http://www.itrs.net.

[4] S. Nelson, et al., "Optimum microstrip interconnects," 1991 IEEE MTT-S Int. Microwave Symp. Dig., pp. 1071-1074, 1991.

[5] H. Y. Lee, "Wideband characterization of a typical bonding wire for microwave and millimeter-wave integrated circuits," IEEE Trans. Microwave Theory and Tech., vol. 43, No. 1, pp. 66-68, Jan. 1995.

[6] A. Wong and D. Linton "Copper flip chip bump interconnect technology for microwave subsystems including RF characterization," Proc. Electronics Packaging Technology Conference, pp. 335 338, 2000

[7] Y. Huang, et al., "Magnetically aligned anisotropic conductive adhesive for high frequency interconnects," 2005 IEEE MTT-S Int. Microwave Symp. Dig. vol.1, pp. 861-864, June 2005.

[8] S. Moon, et al., "Magnetically aligned anisotropic conductive adhesive for microwave applications," IEEE Trans. Microwave. Theory Tech., vol. 56, no.12, pp. 2942-2949, Dec. 2008. 